University of Nebraska - Lincoln

DigitalCommons@University of Nebraska - Lincoln

Faculty Publications in Computer \& Electronics Electrical \& Computer Engineering, Department Engineering (to 2015)

9-2009

\title{
On the Issue of Decoupled Decoding of Codes Derived from Quaternion Orthogonal Designs
}

\author{
Tadeusz Wysocki \\ University of Nebraska-Lincoln, wysocki@uow.edu.au \\ Beata Wysocki \\ University of Nebraska-Lincoln, bwysocki2@unl.edu \\ Sarah Spence Adams \\ Franklin W. Olin College of Engineering, sarah.adams@olin.edu
}

Follow this and additional works at: https://digitalcommons.unl.edu/computerelectronicfacpub

Part of the Computer Engineering Commons

\begin{abstract}
Wysocki, Tadeusz; Wysocki, Beata; and Adams, Sarah Spence, "On the Issue of Decoupled Decoding of Codes Derived from Quaternion Orthogonal Designs" (2009). Faculty Publications in Computer \& Electronics Engineering (to 2015). 61.

https://digitalcommons.unl.edu/computerelectronicfacpub/61
\end{abstract}

This Article is brought to you for free and open access by the Electrical \& Computer Engineering, Department of at DigitalCommons@University of Nebraska - Lincoln. It has been accepted for inclusion in Faculty Publications in Computer \& Electronics Engineering (to 2015) by an authorized administrator of DigitalCommons@University of Nebraska - Lincoln. 


\title{
On the Issue of Decoupled Decoding of Codes Derived from Quaternion Orthogonal Designs
}

\author{
Tadeusz A Wysocki, Senior Member IEEE, Beata J Wysocki, Sarah Spence Adams, Member IEEE
}

\begin{abstract}
Quaternion orthogonal designs (QODs) have been previously introduced as a basis for orthogonal space-time polarization block codes (OSTPBCs). This note will serve to correct statements concerning the optimality of a decoupled maximum-likelihood (ML) decoding algorithm. It will be shown that when compared to coupled decoding, the decoupled decoding is only optimal in certain cases. This raises several open problems concerning the decoding of OSTPBCs.
\end{abstract}

Index Terms - space-time block code, quaternion orthogonal design, decoupled decoding, maximum-likelihood decoding

\section{INTRODUCTION}

A benefit of using complex orthogonal space-time block codes (COSTBCs) [1] over non-orthogonal space-time codes (STCs) [2] is that the orthogonality of the coding matrix allows for a simple maximum likelihood (ML) decoding rule that can be decoupled for every unknown. However, this orthogonality necessarily limits the code's rate, i.e., the ratio of the number of independent complex variables to the number of rows. Liang has shown that full-rate COSTBCs do not exist for more than 2 transmit antennas [3], and he determined that the maximum rate of rectangular COSTBCs approaches $1 / 2$ as the number of columns increases [4]. In particular, Liang showed that for a COSTBC with $2 \mathrm{~m}-1$ or $2 \mathrm{~m}$ columns, the maximum rate is $(\mathrm{m}+1) / 2 \mathrm{~m}$ [4]. This raised the question of whether it is possible to increase the ratio of the number of complex variables to the number of rows by sacrificing orthogonality or by other means. Recently, orthogonal designs over the quaternion domain were considered in part to address this question [5-9].

This introduction of orthogonal designs over the quaternion domain was convenient because quaternions can be viewed as a quaternion combination of complex variables and because they can be used to model rotations. As such, these quaternion orthogonal designs (QODs) over quaternion variables can be used as building blocks for orthogonal space-time-polarization

Tadeusz A Wysocki is with the Department of Computer and Electronics Engineering, University of Nebraska - Lincoln, Peter Kiewit Institute, Omaha, NE 68182-0572 USA, ph. (402) 554-2164 (email: twysocki2@unl.edu).

Beata J Wysocki is with the Department of Computer and Electronics Engineering, University of Nebraska - Lincoln, Peter Kiewit Institute, Omaha, NE 68182-0572 USA.

Sarah Spence Adams is with Franklin W. Olin College of Engineering, Olin Center, Olin Way, Needham, MA 02492-1200, (e-mail: sarah.adams@olin.edu). block codes (OSTBCs) [5-9]. By taking advantage of the view of a quaternion variable as a combination of complex variables, we have shown that it is possible to use QODs to increase the complex rate in a QOD beyond what is possible with a COSTBC. In particular, we will consider in Section III an example of a $2 \times 2$ QOD $\mathbf{Q}$ that achieves a complex rate of $1.25[5]$.

In order to take advantage of this increase in rate, it is necessary to develop an appropriate decoding rule. In fact, Seberry et al. claimed that even though the dual-polarized transmission channel cannot be considered as described by means of a single quaternionic gain, the maximum-likelihood (ML) decoding rule can be decoupled for OSTPBCs derived from QODs [7, Section IV]. Regretfully, decoupled decoding using the method presented therein is only optimal for codes derived from certain QODs, not from arbitrary QODs as previously suggested.

We have attempted to highlight the issue in [10], where we introduced the mathematical notation necessary for correcting the error introduced in [7].

In this paper, we wish to further clarify the problem and explain why the ML decoding rule can only be decoupled for OSTPBCs based on certain QODs, rather than for the general case. Unfortunately, this will imply a problem with the work of other authors [11] who claimed, based on Section IV of [7], that the quaternion orthogonality of an underlying QOD is sufficient to ensure optimal decoupled decoding in the OSTPBC.

To avoid further confusion, we present in this paper an example to demonstrate that the decoupled ML decoding is not always optimal. The example uses an OSTPBC based on the aforementioned $2 \times 2$ QOD $\mathbf{Q}$ with complex rate 1.25. In this example, we will compare the code's performance achieved using the decoupled decoding statistics derived based on decoding rule presented in [7] with the performance of a coupled ML decoding search.

\section{Problem Statement}

In Section IV of our paper [7] dealing with the simulation results, we considered a scenario of $N$ transmit and a single receive-dual polarized antennas as shown in Fig. 1. Hence, each transmission channel is described by the channel gain matrix $\mathbf{H}^{(m)} ; m=1,2, \ldots, N$, where

$$
\mathbf{H}^{(m)}=\left[\begin{array}{ll}
h_{11}^{(m)} & h_{12}^{(m)} \\
h_{21}^{(m)} & h_{22}^{(m)}
\end{array}\right]
$$


where $h_{11}^{(m)}$ and $h_{22}^{(m)}$ are complex channel gains for signals received with the same polarization as they were transmitted, and $h_{12}^{(m)}$ and $h_{21}^{(m)}$ are complex channel gains for a cross-polar scatter, i.e., signals received with different polarization from that at which it was transmitted due to scatter, reflections and polarization twist between the transmit and receive antennas.

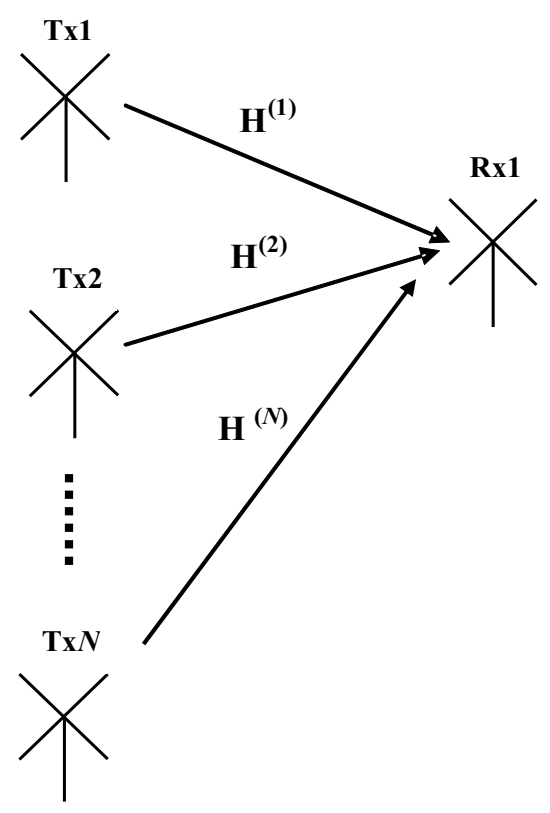

Figure 1: Considered transmission system utilizing $N$ dual-polarized transmit antennas $\mathrm{Tx} 1, \ldots, \mathrm{Tx} N$, and a single receive dual-polarized antenna.

In [7], we have utilized the representation of a quaternion variable $s=z_{1}+z_{2} \mathbf{j}$ as $s=\left[z_{1}, z_{2}\right]$, so that a quaternion matrix $\mathbf{Q}$ can be converted into a complex matrix with twice as many columns $[6,7,9]$. However, we have referred to the complex representation of $\mathbf{Q}$ again as $\mathbf{Q}$ It was possible to use the context (e.g., the implied size or domain) to determine which representation of $\mathbf{Q}$ was being utilized but ultimately lead to a confusion and an abuse of the notation. To the certain extent, we have corrected this problem in [10] by formalizingthe notation, thus illuminating a problem with the decoding discussion in [7, Section IV]. Here, we will further expand on the problem.

Let us define an operator $\mathbb{C}$ from the quaternion to the complex domain such that

$$
\mathbb{C}\left\{z_{1}+z_{2} \mathrm{j}\right\}=\left[\begin{array}{ll}
\mathrm{z}_{1} & \mathrm{z}_{2}
\end{array}\right] \text { and } \mathbb{C}^{-1}\left\{\left[\begin{array}{ll}
\mathrm{z}_{1} & \mathrm{z}_{2}
\end{array}\right]\right\}=z_{1}+z_{2} \mathrm{j}
$$

Now, using the operator $\mathbb{C}$, the received signal component $r^{(m)}$ arriving from the $m$ th transmit antenna that transmits $s$, can be expressed as:

$$
r^{(m)}=\mathbb{C}^{-1}\left\{\mathbb{C}\{s\} \mathrm{H}^{(m)}\right\}+n_{q}
$$

where $n_{q}=n_{1}+n_{2} \mathbf{j}$ and $n_{1}, n_{2}$ are complex noises being the independent identically distributed (i.i.d.) zero-mean two dimensional Gaussian random variables with identical variance per dimension. However, using the notation as in [7, Section IV], the same signal would be given by:

$$
\begin{aligned}
r^{(m)} & =s \mathrm{H}^{(m)}+n_{q} \\
& =\left[z_{1}, z_{2}\right] \mathrm{H}^{(m)}+\left[n_{1}, n_{2}\right] \\
& =\left[z_{1} h_{11}^{(m)}+z_{2} h_{21}^{(m)}, z_{1} h_{12}^{(m)}+z_{2} h_{22}^{(m)}\right]+\left[n_{1}, n_{2}\right] \\
& =\left(z_{1} h_{11}^{(m)}+z_{2} h_{21}^{(m)}\right)+\left(z_{1} h_{12}^{(m)}+z_{2} h_{22}^{(m)}\right) \mathbf{j}+n_{1}+n_{2} \mathbf{j}
\end{aligned}
$$

Then, if an STPBC $\mathbf{Q}$ for $N$ transmit antennas is used, the received dual-polarized signal vector $\mathbf{R}$ can be considered as a quaternionic vector, and modeled as:

$$
\mathrm{R}=\mathbb{C}^{-1}\{\mathrm{C}\{\mathrm{Q}\} \mathrm{H}\}+\mathbf{N}_{\mathrm{q}}
$$

where

$$
\mathrm{H}=\left[\begin{array}{c}
\mathrm{H}^{(1)} \\
\vdots \\
\mathrm{H}^{(N)}
\end{array}\right]
$$

and $\mathbf{N}_{\mathrm{q}}$, is the vector of quaternionic noises being the independent identically distributed (i.i.d.) zero-mean four dimensional Gaussian random variables with identical variance per dimension.

Assuming perfect channel knowledge at the receiver, i.e., assuming that matrices $\mathbf{H}^{(1)}, \ldots, \mathbf{H}^{(N)}$ are known and constant for some reasonable time, as in the case of quasi-static conditions, the maximum likelihood (ML) decoding rule for any STPBC is equivalent to finding a set of signal symbols that minimizes the following quaternion analog of the standard complex Frobenious norm:

$$
\left\|\mathrm{R}-\mathbb{C}^{-1}\{\mathbb{C}\{\mathrm{Q}\} \mathrm{H}\}\right\|
$$

where $\mathbf{R}$ is the received signal vector, $\mathbf{Q}$ is the code matrix, and $\mathbf{H}$ is the matrix of complex channel coefficients.

In Section IV of our paper [7], through an irresponsible abuse of notation, we assumed that this is equivalent to finding a set of signal symbols minimizing the squared norm $\|\mathbf{R}-\mathbf{Q H}\|^{2}$, which, denoting $\{\cdot\}^{Q}$ as quaternion transpose, can be expressed as:

$$
\begin{aligned}
& \|\mathrm{R}-\mathrm{QH}\|^{2}=\operatorname{trace}\left\{(\mathrm{R}-\mathrm{QH})^{Q}(\mathrm{R}-\mathrm{QH})\right\} \\
& \quad=\operatorname{trace}\left\{\left(\mathrm{R}^{Q}-\mathrm{H}^{Q} \mathbf{Q}^{Q}\right)(\mathbf{R}-\mathbf{Q H})\right\} \\
& \quad=\operatorname{trace}\left\{\mathbf{R}^{Q} \mathbf{R}-\mathbf{R}^{Q} \mathbf{Q H}-\mathbf{H}^{Q} \mathbf{Q}^{Q} \mathbf{R}+\mathbf{H}^{Q} \mathbf{Q}^{Q} \mathbf{Q H}\right\} \\
& \quad=\operatorname{trace}\left\{\mathbf{R}^{Q} \mathbf{R}\right\}-2 \operatorname{Re}\left\{\operatorname{trace}\left\{\mathbf{R}^{Q} \mathbf{Q} \mathbf{H}\right\}+\lambda \operatorname{trace}\left\{\mathbf{H}^{Q} \mathbf{H}\right\}\right.
\end{aligned}
$$

since due to orthogonality of $\mathbf{Q}$, we have:

$$
\mathbf{Q}^{Q} \mathbf{Q}=\lambda \mathbf{I}
$$

We have also drawn there a conclusion that for any orthogonal $\mathbf{Q}$, the ML decoding rule can be decoupled.

Unfortunately, neither the expansion of $\|\mathbf{R}-\mathbf{Q H}\|^{2}$ nor the final conclusion about the decoupled ML decoding of orthogonal STPBCs are correct. When the expansion in (6) is done more carefully using the notation in (5), the problem becomes clear. This expansion implicitly assumes that the 
operator $\mathbb{C}$ and its inverse are commutative with the quaternion and Hermitian transposes. Letting $\mathbf{A}=\mathbb{C}\{\mathbf{Q}\} \mathbf{H}$ (a complex product), it was implicitly assumed that $\left[\mathbb{C}^{-1}\{\mathbf{A}\}\right]^{\mathrm{Q}}=\mathbb{C}^{-1}\left\{\mathbf{A}^{Q}\right\}$, or more specifically, that $\left[\mathbb{C}^{-1}\{\mathbf{A}\}\right]^{\mathrm{Q}}=\mathbb{C}^{-1}\left\{\mathbf{A}^{H}\right\}$, as the quaternion transpose simplifies to the Hermitian transpose when acting upon a complex matrix. However, this equation does not hold, as can be seen with the following general example:

$$
\mathbb{C}^{-1}\left\{\left[\begin{array}{ll}
z_{1} & z_{2} \\
z_{3} & z_{4}
\end{array}\right]\right\}^{Q}=\left[z_{1}^{*}-z_{2} \mathbf{j}, \quad z_{3}^{*}-z_{4} j\right]
$$

while

$$
\mathbb{C}^{-1}\left\{\left[\begin{array}{ll}
z_{1} & z_{2} \\
z_{3} & z_{4}
\end{array}\right]^{H}\right\}=\left[\begin{array}{l}
z_{1}^{*}+z_{3}^{*} \mathrm{j} \\
z_{2}^{*}+z_{4}^{*} \mathrm{j}
\end{array}\right]
$$

This nullifies the validity of the expansion in (6). Although the last line in the invalid expansion is now irrelevant, we note for further clarification that the usage there of the orthogonality of $\mathbf{Q}$ would be incorrect, even if it were possible to get to that line. To see this, note that the matrix should be formally written as $\mathbb{C}\{\mathbf{Q}\}$, which is not orthogonal when viewed correctly as a complex matrix.

Therefore, in the general case, the decoupled decoding statistics derived using (6) do not lead to ML decoding. To achieve the ML decoding rule, one needs to minimize the norm given by (5), which can only lead to decoupled decoding in special cases, like the case of an example considered in [7].

\section{Simulation Results}

In this section, we describe the simulation results of an OSTPBC to further illustrate the issues concerning coupled versus decoupled decoding rules. Let us consider the QOD of order 2 given by the matrix $\mathbf{Q}$ :

$$
\mathbf{Q}=\left[\begin{array}{lr}
\left(x_{3}+x_{4} \mathbf{i}\right)+\left(x_{0}+x_{2} \mathbf{i}\right) \mathbf{j} & \left(x_{3}+x_{4} \mathbf{i}\right)+\left(x_{1}+x_{2} \mathbf{i}\right) \mathbf{j} \\
\left(x_{3}+x_{4} \mathbf{i}\right)-\left(x_{1}-x_{2} \mathbf{i}\right) \mathbf{j} & \left(-x_{3}-x_{4} \mathbf{i}\right)+\left(x_{0}-x_{2} \mathbf{i}\right) \mathbf{j}
\end{array}\right]
$$

where $x_{0}, x_{1}, x_{2}, x_{3}, x_{4}, x_{5}$ are real variables. Using quaternionic arithmetic, it is easy to prove that $\mathbf{Q}$ is orthogonal. Then, utilizing the operator $\mathbb{C}, \mathbf{Q}$ can be reexpressed as:

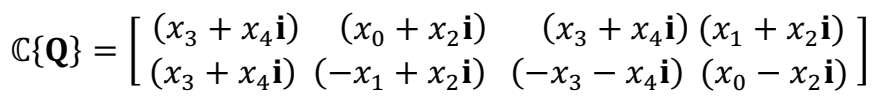

and used to transmit 5 real variables in 2 consecutive time slots through 2 transmit dual-polarized antennas.

If the system uses a single receive dual-polarized antenna, then the ML decoding is equivalent to finding a set of signal symbols that minimizes the following norm:

$$
\left\|\mathrm{R}-\mathbb{C}^{-1}\{\mathbb{C}\{\mathrm{Q}\} \mathrm{H}\}\right\|
$$

where $\mathbf{R}$ is the received quaternion signal vector, $\mathbf{R}^{Q}=\left[r_{1}^{Q}, r_{2}^{Q}\right]$, and $\mathbf{H}$ is the $4 \times 2$ matrix of channel gain coefficients:

$$
\mathbf{H}=\left[\begin{array}{ll}
h_{11}^{(1)} & h_{12}^{(1)} \\
h_{21}^{(1)} & h_{22}^{(1)} \\
h_{11}^{(2)} & h_{12}^{(2)} \\
h_{21}^{(2)} & h_{22}^{(2)}
\end{array}\right]
$$

Such a decoding can be performed searching through all the possible fivetuplets $\left(x_{0}, x_{1}, \ldots, x_{4}\right)$ taking elements from the transmit symbol alphabet $\mathbf{X}$. For a low order modulation, like QPSK, this means checking $2^{5}=32$ combinations for every block of 5 received symbols.

Assuming the same simulation conditions as in [7], the performance of a system using the OSTPBC given by (6) and (7) with the coupled ML decoding is excellent, and shown in Fig. 2.

For $x_{0}, x_{1}, x_{2}, x_{3}, x_{4} \in \mathbf{X}$, where $\mathbf{X}$ is the set of all possible amplitudes in the multilevel QAM signal, the decoupled decoding rules derived from (5), are as follows:

- Decoding for $x_{0}$, is finding $x_{0} \in \mathbf{X}$ that minimizes:

$\left|x_{0}\right|^{2} \sum_{m, n, l=1,2}\left|h_{m n}^{(l)}\right|^{2}-2 x_{0} \operatorname{Re}\left\{r_{1}^{Q}\left(h_{21}^{(1)}+h_{22}^{(1)} \mathbf{j}\right)+r_{2}^{Q}\left(h_{21}^{(2)}+h_{22}^{(2)} \mathbf{j}\right)\right\}$

- Decoding for $x_{1}$, is finding $x_{1} \in \mathbf{X}$ that minimizes:

$\left|x_{1}\right|^{2} \sum_{m, n, l=1,2}\left|h_{m n}^{(l)}\right|^{2}-2 x_{1} \operatorname{Re}\left\{r_{1}^{Q}\left(h_{21}^{(2)}+h_{22}^{(2)} \mathbf{j}\right)-r_{2}^{Q}\left(h_{21}^{(1)}+h_{22}^{(1)} \mathbf{j}\right)\right\}$

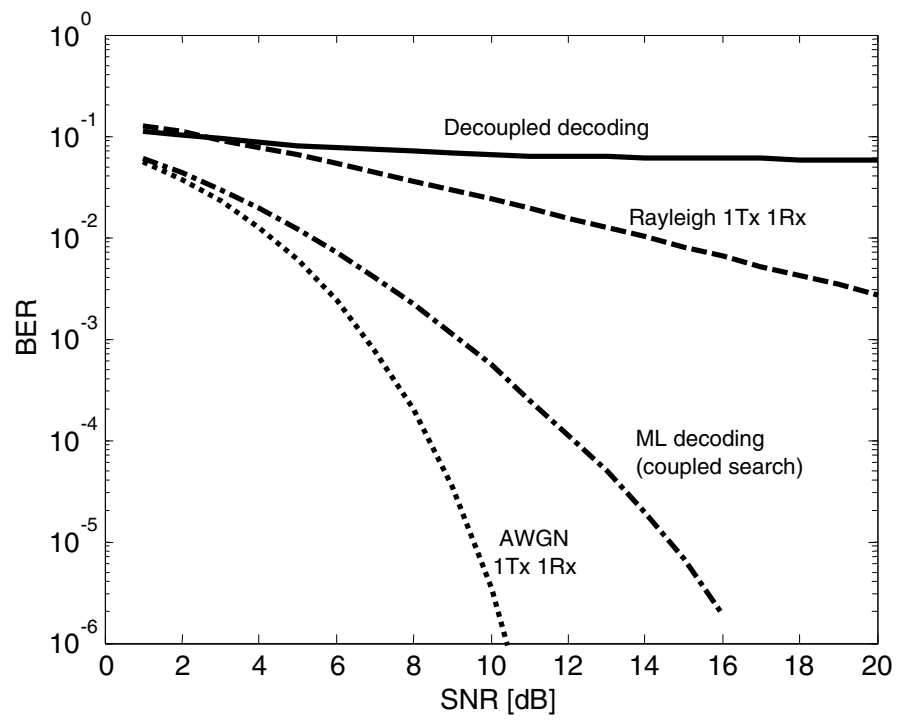

Figure 2: Bit-error-rate (BER) performance of the considered OSTPBC combined with QPSK modulation in a slow Rayleigh fading channel experiencing random cross-polar scatter using the coupled ML decoding and decoupled decoding.

- Decoding for $x_{2}$, is finding $x_{2} \in \mathbf{X}$ that minimizes:

$$
\begin{aligned}
& 2\left|x_{2}\right|^{2} \sum_{m, n, l=1,2}\left|h_{m n}^{(l)}\right|^{2}-2 x_{2} \operatorname{Re}\left\{r_{1}^{Q} \mathbf{i}\left[\left(h_{21}^{(1)}+h_{21}^{(2)}\right)+\left(h_{22}^{(1)}+h_{22}^{(2)}\right) \mathbf{j}\right]\right. \\
& \left.\quad+r_{2}^{Q} \mathbf{i}\left[\left(h_{21}^{(1)}-h_{21}^{(2)}\right)+\left(h_{22}^{(1)}-h_{22}^{(2)}\right) \mathbf{j}\right]\right\}
\end{aligned}
$$

- Decoding for $x_{3}$, is finding $x_{3} \in \mathbf{X}$ that minimizes: 


$$
\begin{aligned}
& 2\left|x_{3}\right|^{2} \sum_{m, n, l=1,2}\left|h_{m n}^{(l)}\right|^{2}-2 x_{3} \operatorname{Re}\left\{r_{1}^{Q}\left[\left(h_{11}^{(1)}+h_{11}^{(2)}\right)+\left(h_{12}^{(1)}+h_{12}^{(2)}\right) \mathbf{j}\right]\right. \\
& \left.\quad+r_{2}^{Q}\left[\left(h_{11}^{(1)}-h_{11}^{(2)}\right)+\left(h_{12}^{(1)}-h_{12}^{(2)}\right) \mathbf{j}\right]\right\}
\end{aligned}
$$

- Decoding for $x_{4}$, is finding $x_{4} \in \mathbf{X}$ that minimizes:

$$
\begin{aligned}
& 2\left|x_{4}\right|^{2} \sum_{m, n, l=1,2}\left|h_{m n}^{(l)}\right|^{2}-2 x_{4} \operatorname{Re}\left\{r_{1}^{Q} \mathbf{i}\left[\left(h_{11}^{(1)}+h_{11}^{(2)}\right)+\left(h_{12}^{(1)}+h_{12}^{(2)}\right) \mathbf{j}\right]\right. \\
& \left.+r_{2}^{Q} \mathbf{i}\left[\left(h_{11}^{(1)}-h_{11}^{(2)}\right)+\left(h_{12}^{(1)}-h_{12}^{(2)}\right) \mathbf{j}\right]\right\}
\end{aligned}
$$

It should be noted here that albeit channel gain coefficients are complex numbers, operations within $\operatorname{Re}\{\cdot\}$ operator must be performed using quaternionic arithmetic. The decoupled decoding rules are much simpler to implement as in the QPSK case they involve just checking $2 \times 5=10$ possibilities for every block of 5 received symbols.

Unfortunately, as it can be seen from Fig.2, the performance of the decisions is far from ML quality.

\section{CONCLUSIONS}

By introducing a more formal mathematical notation and by analyzing the decoding rules of a specific OSTPBC, we have shown that the decoupled decoding rule for orthogonal spacetime-polarization block codes (OSTPBCs) given in [7] is not correct for a general case of OSTPBC. Therefore, finding either a general decoupled decoding ML rule for OSTPBCs or a semi-optimal decoupled rule giving good performance remains an open research question. We hope that further study of the mathematical properties of the available valid examples will lead to more specific guidelines for which QODs can be used to build OSTPBCs that enjoy an optimal decoupled ML decoding rule.

\section{REFERENCES}

[1] V.Tarokh, H.Jafarkhani, A.R.Calderbank: "Space-Time Block Codes from Orthogonal Designs," IEEE Trans. on Info. Th, Vol. 45, No. 5, pp. 1456-1467, 1999.

[2] N.Seshardi, V.Tarokh, and A.R.Calderbank: "Space-time codes for high data rate wireless communications: Code construction," IEEE VTC'97, pp.637-641.

[3] X.-B.Liang, and X.G. Xia: "On the Nonexistence of Rate-One Generalized Complex Orthogonal Designs", IEEE Trans. Information Theory, Vol.49, no.11, pp.2984-2988, 2003.

[4] X.-B.Liang: "Orthogonal designs with maximal rates," IEEE Trans. Information Theory, vol.49, no.10, pp.2468-2503, 2003.

[5] T.A Wysocki, J. Seberry, and B.J. Wysocki: "Space-Time-Polarization Block Codes from Quaternionic Orthogonal Designs," - invited presentation, 9th International Symposium on Communications Theory and Applications, ISCTA’2007, Ambleside, UK, 16-20 July 2007.

[6] B. J Wysocki, T. Wysocki, J. Seberry, S. S. Adams, H. Sharif: "A Simple Orthogonal Space-Time-Polarization Block Code", IEEE VTC'2007-Fall, 1-3 October 2007, Baltimore, USA.

[7] J.Seberry, K.Finlayson, S. Spence Adams, T.A Wysocki, T.Xia, and B.J.Wysocki: "The Theory of Quaternion Orthogonal Designs," IEEE Trans. on Signal Processing, Vol.56, No.1, pp.256-265.

[8] S. S. Adams, J. Seberry, N. Karst, J. Pollack, T. Wysocki: "Quaternion Orthogonal Designs from Complex Companion Designs," Linear Algebra and its Applications, Vol. 428, Issue 4, pp. 1056-1071, Feb 2008.

[9] B. J Wysocki, T. Wysocki, S. S. Adams: "On an Orthogonal SpaceTime-Polarization Block Code", Journal of Communications, to appear.

[10] Errata

[11] H. Ma, Q. Lan, H. Kan, H. Imai, "A Novel Quaternion Design Construction For STBC," IEEE International Conference on Communications, 2008 (ICC '08), pp. 2791-2795, May 2008. 